\title{
ZugANG ZUR MACHT. WEGE ZUM HERRSCHER
}

\author{
Christiane Kunst
}

Universität Osnabrück

ZuSAMMENFASSUNG: Im Verlauf seiner Herrschaft inszenierte Alexander seine Person in Anlehnung an das achämenidische Herrscherzeremoniell. Der Zugang zum König, gerade bei Audienzen, wurde immer stärker reglementiert und einem festgelegten Protokoll unterworfen. Der differenzierte Zugang zum König kennzeichnete den Rang eines Individuums im Reich. Alexander übernahm dabei Traditionen der Distanzierung des persischen Königs, bediente sich gleichzeitig aber neuer Formen, indem er seine Gefährten zu menschlichen Sichtschranken und Schutzschilden erhob. Diesen Weg sind die hellenistischen Könige weitergegangen, allerdings nicht ohne gegenüber den Poleis nach den Regeln des Theaters zeremoniell festgelegte Auftritte in der Öffentlichkeit zu inszenieren, und so im traditionellen griechischen Herrschaftsverständnis wurzelnde Bedürfnisse nach Autonomie zu befriedigen.

Schlüsselbegriffe: Alexander der Große, Hofzeremoniell, Hellenismus, Monarchie, Repräsentation.

ABSTRACT: In the course of his reign, Alexander fashioned his royal persona by making use of Achaimenid court protocol. Access to the king, especially at the time of audiences, was regulated ever more strictly and governed by a rigid set of rules. The graded access to the king marked an individual's rank in the empire. Alexander took over certain traditions of distancing from the Persian kings, while simultaneously establishing new forms by elevating his companions to specific functions, for example as human barriers against sight or human shields. Later, Hellenistic kings followed this route, but not without carefully modelling the ceremonial 
of their public appearances in the Greek poleis along the rules of the theatre. In so doing, they satisfied the need for autonomy which was so deeply rooted in traditional Greek notions of government.

Keywords: Alexander the Great, court protocol, Hellenism, monarchy, representation.

B ei Herodot lesen wir, wie der Mederkönig sich mit den Magiern über die reale Gefahr berät, die vom Knaben Kyros für das eigene Reich ausgehen könnte. Kyros, heißt es, habe sich zum König der Dorfjugend aufgeschwungen: „Und er hat alles so gerichtet, wie Könige es tun. Denn er hat Lanzenträger (d.h. Wächter) und Türhüter und Botschaftsträger und sonst alles eingeteilt und hat regiert". ${ }^{1}$ Monarchie wird durch die Existenz eines „Hofstaats“, sowie die räumliche Distanzierung von Herrscher und Beherrschten definiert. An anderer Stelle beschreibt Herodot $(1,99)$, wie der Mederkönig seine ta oikía (gemeint ist wohl so etwas wie ein privater Wohnbereich) mit einer Mauer umgab, um die herum er das übrige Volk siedeln ließ, also außerhalb des Palastgeländes. Daran anschließend erläutert der Autor die vom König eingeführte Hofordnung der Kommunikation: Niemand wird zum König vorgelassen; Entscheidungen und Beschlüsse werden ausschließlich durch Boten mitgeteilt. Der König soll häufiges Gesehenwerden vermeiden und auf diese Weise seine Herausgehobenheit und Ferne inszenieren. Die Abgeschiedenheit verschafft dem König zudem Sicherheit.

Die Griechen des 5. Jhs. nahmen ihre eigene Vorstellung von Herrschaft in Abgrenzung zu tatsächlichen oder angeblichen orientalischen Konzepten wahr. Das galt auch für monarchische Herrschaften in Hellas. Xenophon (9,12) äußert in seiner Lobrede auf den Spartanerkönig Agesilaos II. (398-361 v.Chr.):

Jch will aber auch angeben, wie er in seiner Lebensweise einen Gegensatz bildete zu der Hoffart des Perserkönigs. Erstens nämlich setzte dieser eine Ehre darein sich selten sehen zu lassen Agesilaos aber hatte seine Freude daran imner sichtbar zu sein, denn er meinte der Schlechtigkeit stehe es an, sich den Augen der Leute zu entziehen dem tngendhaften Leben aber verleihe das Licht vielmehr Glanz. Dann hielt jener es für eine Ehre schwer zugänglich zu sein, ihm [Agesilaos] aber machte es Vergnügen, allen leichten Zugang zu gewähren

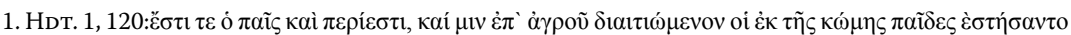

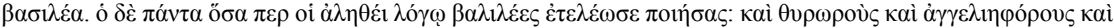

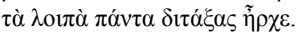


und Jener [der Perserkönig] suchte etwas darin, langsam seine Geschäfte zu beenden, ihm [Agesilaos] aber machte es am meisten Freude, wenn er so schnell als möglich den Leuten, was sie baten gewähren und sie entlassen konnte. ${ }^{2}$

Leutseligkeit und Präsenz des Spartanerkönigs werden mit einer düsteren Zurückgezogenheit des persischen Monarchen kontrastiert. Historisch fassbar wird der Gedanke der räumlichen Distanzierung in der griechischen Welt zuerst in der jüngeren Tyrannis, als Dionysios von Syrakus (405-367 v.Chr.) seine Residenz auf der Halbinsel Ortygia errichtete, umgeben von den Häusern seiner Vertrauten. ${ }^{3}$

Von Alexander dem Großen wissen wir, dass er spätestens nach dem Erfolg von Issos 333 v.Chr. das Reich des Dareios III. (336-331 v.Chr.) anstrebte und sich nach dessen Tod 330 als Erbe des Achäemenidenherrschers inszenierte. ${ }^{4}$ Ein wichtiges Element der Repräsentation des orientalischen Königs war seine Distanzierung. ${ }^{5}$ Untersucht werden soll daher, wie der Zugang zum Herrscher, der Weg zur Macht, durch Alexander und seine Nachfolger geregelt wurde.

\section{Wege Zu Alexander}

Schon vor seinem Einzug in Mesopotamien und in der Persis hat Alexander erkennen lassen, dass er Konzepte des orientalischen Königtums für erstrebenswert hielt. Beim Entwurf des Stadtplans von Alexandria plante er so Diodor $(17,52)$ - seinen Palast auf der Halbinsel Lochis, also zurückgezogen vom üblichen Stadtareal. ${ }^{6}$ Dass darin mehr als ein Sicherheitskonzept steckt, verdeutlichen Alexanders parallele Bemühungen, sukzessiv Formen des achäemenidischen Hofes zu adaptieren.

Kurzer Rückblick: Alexanders Hof war in der Zeit von seiner Abreise aus Pella bis zu seinem Tod in Babylon einer grundlegendenden Transformation unterworfen gewesen. Jahrelang hatte er aus einem mobilen Feldlager regiert und erst 323 nach seiner Rückkehr aus Indien nutzte Alexander die neubabylonische Palastanlage in Babylon als Herrschaftszentrale. In Folge seiner außergewöhnlichen militärischen Erfolge und großer Beute war es ihm im Verlauf des Feldzugs gelungen, sich selbst zur unangefochtenen Mitte

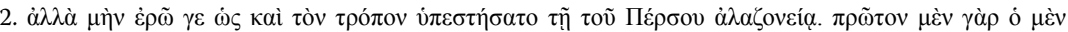

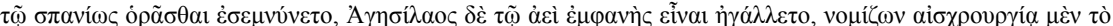

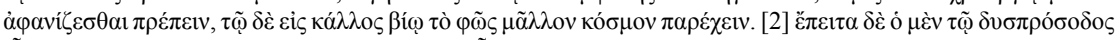

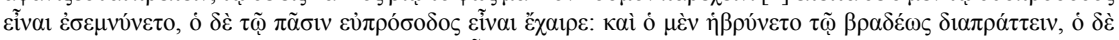

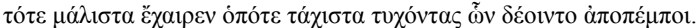

3. K. F. Stroheker: Dionysios I. Gestalt und Geschichte des Tyrannen von Syrakus, Stuttgart 1958, 157.

4. H.-W. Rit TER: Diadem und Königsherrschaft. Untersuchungen zu Zeremonien und Rechtsgrundlagen des Herrschaftsantritts bei den Persern, bei Alexander dem Großen und im Hellenismus, München 1965, p. 53f; E.A. Fredericksmeyer: „Alexander the Great and the Kingdom of Asia“, in: A.B. Bosworth - E.J. Baynham (Hg.): Alexander the Great in Fact and Fiction, Oxford 2000, 136-166.

5. L. Llewellyn-Jones: King and Court in Ancient Persia 559 to 331 BCE, Edinburgh 2013, 42ff.

6. Vgl. J., McKenzie: The Architecture of Alexandria and Egypt, c. 300 B.C. to A.D. 700, Yale 2007, 38. 
seiner Herrschaft zu machen und konkurrierende Kräfte auszuschalten oder weitgehend zu marginalisieren. ${ }^{7}$ Diese im Vergleich zum makedonischen König neue Stellung fand in einer zunehmenden Zeremonialisierung der Herrschaft ihren Niederschlag. Hierzu gehörten die Einführung von Hofämtern sowie eine strukturierte Regelung des Zugangs zum König. Der König begann sich zu entziehen; er war nicht länger für jeden direkt erreichbar. Die Chronologie der Distanzierung ist schwierig nachzuvollziehen.

Ein erstes Mittel, um den Zugang zum König zu strukturieren, bot Dareios rotes Prachtzelt, das Alexander nach dem Erfolg von Issos zusammen mit entsprechenden Bediensteten in die Hände gefallen war und repräsentative Funktionen übernahm. ${ }^{8}$ Spätestens ab 330 v.Chr., nach dem Tod des Dareios, mussten sich auswärtige Gesandte an den eisangeleus wenden, um zum König vorgelassen zu werden. Als erster scheint Chares von Mytilene mit diesem Amt betraut worden zu sein. ${ }^{9}$

329 führte Alexander mit der Übernahme von Kleidungsstücken des Dareios auch ein Zugangsprotokoll ein. Diodor schreibt:

Als erster richtete er asiatischstämmige Stabträger (rhabdouchous Asiageneis) an seinem Hof ein, dann beorderte er die ausgezeichnetsten der Männer als seine Wachen (doryphorein), unter denen auch der Bruder des Dareios, Oxyathres, war...[legt persische Tracht an]...darauf verteilte er purpurgesäumte Gewänder an seine Hetairoi. ${ }^{10}$

Die hier erwähnte Garde der Lanzenträger, von den griechischen Historiographen auch Melophoroi genannt, hatte den inneren Bereich des königlichen Palasts des Großkönig gesichert. Nun schuf Alexander eine "neue" persische Wache für sich unter persischen Kommandeuren. ${ }^{11}$ Gleichzeitig wurde Purpur als Trennfarbe an die Vertrauten, vermutlich die engeren Hetairoi ausgegeben, ${ }^{12}$ in deren Kreis auch Angehörige der Alten Führungsschicht wie Oxyathres einbezogen wurden. ${ }^{13}$

Im Winterquartier von Bakra 329/28 unternahm Alexander den Versuch durch Einführung der Proskynese, die nicht länger aus Makedonen bestehende engere Elite auf ein einheitliches (persisches) Ritual der Begrüßung zu verpflichten. Die Proskynese fungierte für den Perserkönig als Instrument seiner Entrückung: Eine festgelegte Handlungskette ${ }^{14}$ festigte dabei die Position

7. G. Weber: „Der Hof Alexanders des Großen als soziales System“, Saeculum 58, 2007, 229-264.

8. Plut. Alex. 20.

9. Plut. Alex. 46, 2.

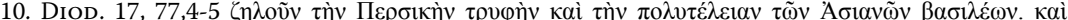

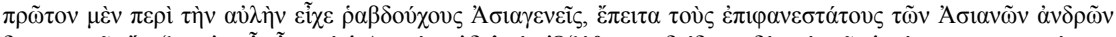

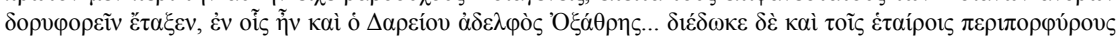
$\sigma \tau 0 \lambda \grave{\alpha} \zeta \ldots$

11. Arr. 7, 11. H. Blum: Purpur als Statussymbol in der griechischen Welt, Bonn, 1998.

12. DiOD. $17,77,4-5$.

13. G. Weber: „Der Hof Alexanders des Großen als soziales System“, Saeculum 58, 2007, 229-264, 242.

14. R. Rollinger: „Herrscherkult und Königsvergöttlichung bei Teipiden und Achaimeniden. Realität 
des Großkönigs zwar nicht als Gott - wie die Griechen und Makedonen die Ereignisse deuteten ${ }^{15}$ - sondern als vom Gott Ahuramazda mit seiner Macht Ausgezeichneter. Trotz Alexanders Angeboten, den Makedonen und Griechen die Annahme der Proskynese durch allein ihnen zugestandene Gesten der Egalität und höfischen Exklusivität schmackhaft zu machen ${ }^{16}$, scheiterte der König. Der König zerfiel in diejenigen, die die Proskynese vollzogen und jene, die das nicht taten.

Gleichzeitig konnte der Verzicht auf die Proskynese gegenüber Griechen und Makedonen jedoch nicht darüber hinwegtäuschen, dass der Zugang zum König strengen Regeln unterworfen war. Ausdruck dessen war das königliche Prunkzelt. Um zum Monarchen zu gelangen, waren die langen Reihen seiner Eliteeinheiten $\mathrm{zu}$ durchschreiten. Die ganze Machtfülle Alexanders entfaltete sich in der zur Schau gestellten Pracht waffenstarrender Kämpfer. Demgegenüber behauptete der Historiker Phylarchos ${ }^{17}$ machten die materiellen Zeichen von Pracht, wie die goldenen Platanen und mit kostbarsten Edelsteinen verzierten Rebstöcke, unter denen die Perserkönige Hof gehalten und ihre Staatsgeschäfte abgewickelt hatten,

einen geringeren Eindruck als der Aufwand, der täglich an Alexanders Hof zu allen Gelegenheiten betrieben wurde. Denn dessen Zelt hatte Platz für einhundert Klinen und wurde von fünfzig goldenen Masten gestützt. Oben bildeten die sich darüber spannenden goldenen Himmelsdarstellungen, die mit prächtigen Mustern gearbeitet waren, das Dach. Als erste Reihe standen fünfhundert ,Apfelträger' (melophoroi) an der Innenwand darum herum, in purpurne und apfelfarbene [gold?] Uniformen gekleidet!" danach eintausend Bogenschützen (toxotai), die einen in feuerroter Kleidung, die anderen in scharlachroter, viele hatten aber auch dunkelblaue Umhänge. An deren Spitze standen ferner fünfhundert Makedonen mit silbernen Schilden (Argyraspides). In die Mitte des Zeltes wurde ein goldener Thron gestellt, auf dem Alexander saß und die Amtshandlungen vornahm, während die Leibwächter auf allen

oder Fiktion?", in: L.-M GÜNTHER - S. PlisChKe (Hg.): Studien zum vorhellenistischen und hellenistischen Herrscherkult, Berlin, 2011 (Oikumene. Studien zur antiken Weltgeschichte 9), 11-54, bes. pp.23ff siehe auch A. ZgOLL: „Audienz - Ein Modell zum Verständnis mesopotamischer Handerhebungsrituale“, Baghdader Mitteilungen 35, 2003, 182-203.

15. HDt. 7,136; Plut. Them. 27,4.

16. ARr. $4,12,3$

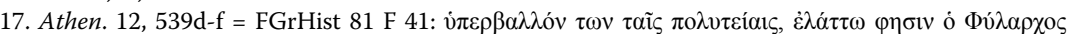

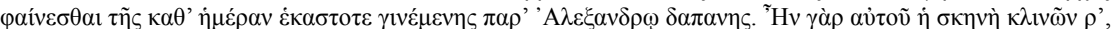

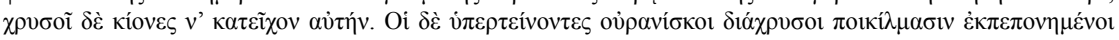

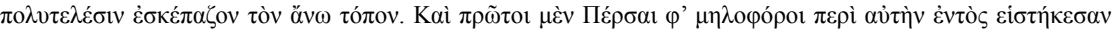

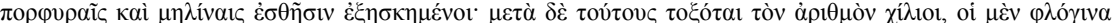

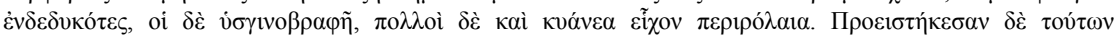

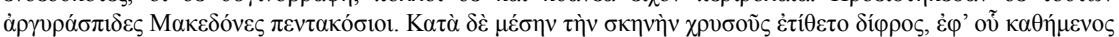

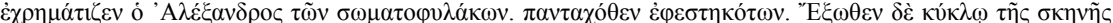

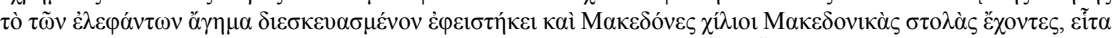

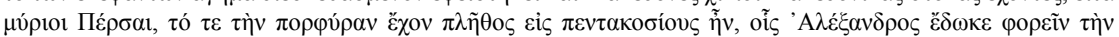

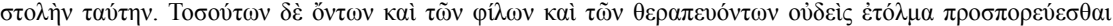

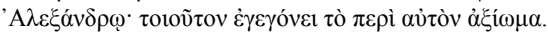


Seiten dabeistanden. Außerhalb des Zeltes hatte der Elefantentrupp und eintausend Makedonen in makedonischer Tracht Aufstellung genommen, ferner zehntausend Perser und die übrige Menge, die in Purpur gekleidet war; sie belief sich etwa auf fünfhundert Menschen. Ihnen hatte Alexander das Anlegen dieser Kleidung als Auszeichnung verliehen. Da aber die Anzahl seiner Freunde und Gefolgsleute so groß war, wagte es keiner, sich Alexander zu nähern. So hoch war die Wertschätzung, die man seiner Person entgegenbrachte. ${ }^{18}$

Das prächtige Zelt bot nur den äußeren Rahmen, entscheidend war die Zurschaustellung der Militärmacht, und die schiere Menge des fein ausdifferenzierten Gefolges - gestaffelt nach Makedonen und Persern. Niemand wagte demnach unaufgefordert sich dem König zu nähern. Diese militärisch-zivile Elite umgab ihn wie ein lebender Schutzschild. Im Inneren des Zelts schirmten seine persisch-makedonischen Gardetruppen den König ab, außen hatten weitere Eliteeinheiten Aufstellung genommen. Das traditionelle persische Zugangszeremoniell, das auf den Apfelträgern beruhte, die aus den zehntausend besten Persern, den sogenannten Unsterblichen, ausgewählt waren, ${ }^{19}$ wurde erweitert um die makedonischen Einheiten der Silberschildner. Den innersten Kreis bildeten die sieben Leibwächter Alexanders, die in Babylon als 24-Stunden-Ordonanz fungierten und den Pagenchor befehligten, der dem König unmittelbar aufwartete, wozu beispielsweise auch das Schlafen in seiner Kammer gehörte. ${ }^{20}$

Die Purpurkleidung grenzte den engeren Kreis um Alexander ab. Sie war Auszeichnung und äußeres Zeichen königlicher Gunst. Es wird aber auch deutlich, dass der Kreis der Purpurträger die zur Verfügung stehende Zahl an Speisebetten überstieg. Zwischen 80 und 100 Hetairoi wurden zur Tafel zugelassen. Das stand in deutlichem Gegensatz zur großen Zahl der Gäste des Großkönigs. Seine Hetairoi bewirtete der König jedoch mit einer festgelegten Summe, die pro Person den Aufwendungen der persischen Tafel entsprach. ${ }^{21}$ Allein die sieben somatophylakes scheinen freien Zugang zum König gehabt zu haben. Sie verkörperten ein Höchstmaß an Ehre und Treue. ${ }^{22}$ Beides hatten sie aufgrund ihres vorbildlichen Verhaltens unter Beweis gestellt und waren

18. Ein an Alexanders Leichenwagen angebrachtes Fries, das den König auf einem Wagen zeigte, bildete eine vergleichbare Entourage ab: „Von diesen zeigte die erste [Bildtafel] einen Wagen von getriebener Arbeit und Alexander mit einem prächtigen Szepter darauf sitzend; um den König befand sich ein Gefolge von Makedonen in voller Rüstung und ein anderes von persischen Apfelträgern und vor diesen Waffenträger“.

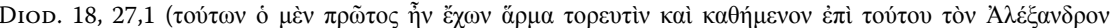

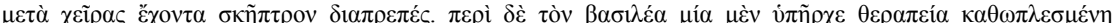

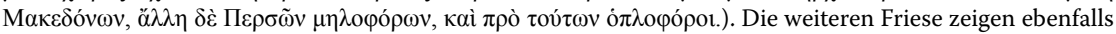
Waffengattungen und müssen entsprechend in Analogie zur Aufstellung vor dem Prunkzelt betrachtet werden.

19. Herakleides von Kyme in Persika Athen. 12, 514c.

20. Bei anderen offiziellen Gelegenheiten sitzt er auf dem Thron, während seine Freunde auf Bänken mit silbernen Füßen ihn umgaben vgl. Arr. 7, 24.

21. Vgl. Athen 4, 146.

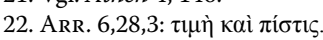


entsprechend von Alexander durch besonders wertvolle Geschenke und Auszeichnungen geehrt worden. Der freie Zugang zum König war Ausdruck von wechselseitiger Treue und Vertrauen. Diodor $(17,61,3)$ nennt Hephaistion ihren Anführer. Spätestens in Susa, als dieser 324 zum Chiliarchen ernannt wurde und damit zumindest formal Oberkommandant der Garde wurde, kann von einer solchen Vorrangstellung die Rede sein. Der Titel Chiliarch brachte den höchsten Hofrang des Hephaistion zum Ausdruck. Dazu gehörte es in persischer Tradition, die Aufgaben eines Protokollchefs wahrzunehmen, ${ }^{23}$ was ihm tatsächlich einen Vorrang vor den übrigen Leibwächtern eingeräumt hätte. Alexander hat ihn jedoch tatsächlich als Stellvertreter behandelt, ${ }^{24}$ so dass Hephaistion im doppelten Sinn dem Thron am nächsten stand.

Nähe und Zugang zu Alexander waren von entscheidender Bedeutung für die Definifion von Rang und Status jedes Individuums. In diesem Kontext fiel der sogenannten. Massenhochzeit von Susa im Frühjahr 324 v.Chr. die entscheidende Bedeutung bei der Neuordnung der Hofelite zu. Angehörige der alten Aristokratie des Perserreichs wurden in der für sie traditioneller Form der Eheschließung, die für die Makedonen ebenfalls verständlich und nachvollziehbar war, in den Hof integriert. der makedonischen Gruppe von Hetairoi wurden auf diese Weise nach persischem Vorbild die ,Verwandten' (syngeneis) des Königs zur Seite gestellt. Der Hof bestand folglich aus den Freunden (engeren Hetairoi), den Verwandten des Königs und den Verwandten der Freunde (des Königs). ${ }^{25}$ Hephaistions herausgehobene Stellung wird durch die Brautwahl in Susa erneut bestätigt, durfte er doch Alexanders Schwägerin heiraten, eine persische Königstochter. ${ }^{26}$

Den durch Freundschaft und Verwandtschaft ausgezeichneten Männern gestattete Alexander, anders als die persischen Könige, ein Höchstmaß an persönlicher Nähe. Alexander aß nicht getrennt von den Getreuen, sondern mit diesen gemeinsam. ${ }^{27}$ Wurde die Tischgemeinschaft hingegen ausgeweitetet, zeichnete sich eine Rangfolge ab. ${ }^{28}$ Nach dem Essen ließ er den goldenen Becher kreisen. ${ }^{29}$ Er beriet sich mit den Gefährten, aß und trank mit ihnen. Eigens für diese veränderte soziale Praxis scheinen bauliche Veränderungen in Babylon am Palast vorgenommen worden zu sein. ${ }^{30}$ Die Aulè des Königs, ein

23. Plut. Them. 27; L. Llewellyn-Jones: King and Court in Ancient Persia, 559 to 331 BC, Edinburgh, 2013, 43.

24. A.W. Collins: „The office of chiliarch under Alexander and the successors“, Phoenix, 55, 2001, 259-283.

25. H. Volkmann: Der Zweite nach dem König, Philologus, 92, 1937, 285-316.

26. ARr. $7,4,5$

27. Perserkönig speist in der Regel allein mit Mutter und Gattin vgl. Plut. Artoxerx. 5,5, während die Gäste je nach Rang in seiner Nähe Mahl halten vgl. Athen. 4, 145a (Herakleides von Kyme).. Zu Alexander vgl. E.N. Borza, „The Symposium at Alexander's Court“, Ancient Macedonia, 3, 1983, 45-55.

28. ARr. 7,11.

29. ARr. $4.12,13$.

30. B. FunCK, „Beobachtungen zum Begriff des Herrscherpalastes und seiner machtpolitischen Funktion im hellenistischen Raum. Prolegomena zur Typologie der hellenistischen Herrschaftssprache“, in: G. Brands, - W. Hoepfner (Hg): Basileia. Die Paläste der hellenistischen Könige, Mainz, 1996, 44-55. 
Begriff der sich bei Arrian gerade im Kapitel 7,25 häuft, wird zum Zentrum des Hofs ${ }^{31}$ und Höfe zum zentralen Merkmal hellenistischer Hofarchitektur. ${ }^{32}$

\section{Hellenistische MonarChen}

Entgegen der landläufigen Meinung sind die hellenistischen Monarchen Alexander stärker gefolgt, als dass sie seiner Politik der Inszenierung der Majestät den Rückken gekehrt hätten. Das wird besonders deutlich beim Blick auf Kleomenes von Sparta (235 und 222 v. Chr.). Plutarch (Kleom. 13) berichte:

Denn, wenn die Menschen mit den anderen Königen [als Kleomenes] zu tun hatten, so waren sie nicht so betroffen von ihrem Reichtum und Glanz, wie sie sich abgestoßen fühlten durch ihren Stolz und Hochmut, wenn sie den bei ihnen Vorsprechenden schroff und verächtlich begegneten. Wenn sie hingegen zu Kleomenes kamen, der doch auch ein König war und so genannt wurde, und bei ihm keine Purpurmmäntel oder Prachtgewänder, keine kostbaren Ruhebetten noch Tragsessel zu sehen bekamen, wenn sie weiter sahen, daß er nicht durch einen Schwarm von Boten und Türhütern noch auf schriftlichem Wege in demütigender Weise allenfall seine Bescheide erteilte, sondern selbst in einfachem Kleid zur Begrüßung kam und da mit denen, die etwas wünschten, heiter und freundlich sprach und für sie Zeit hatte, so wurden sie bezaubert und ganz für ihn gewonnen und sagten, dieser allein sei der wahre Abkömmling des Herakles. ${ }^{33}$

Kleomenes, das liegt auf der Hand, pflegte einen anderen Lebens- und Herrschaftsstil als die anderen Könige des Hellenismus. Wir haben es freilich mit einem hochgradig konstruierten Bild zu tun. Dennoch wird deutlich: der Weg zur Macht wurde in den hellenistischen Monarchien - oder sollte nur Philipp V. gemeint sein - von Hindernissen verstellt. Verschiedene Aspekte sind erkennbar. Die Palastarchitektur, die Ausstattung dienten der Beeindruckung der Beherrschten, aber auch der Entrückung des Königs. Der Monarch begrenzte den Zugang zu seiner Person, bediente sich Boten bzw. der Schriftform, um Entscheidungen mitzuteilen. War er auf dem Thron sichtbar, wenn man zur persönlichen Audienz zugelassen wurde, entzog er sich erneut

31. Ausführlich B. FUNCK: „Beobachtungen zum Begriff des Herrscherpalastes und seiner machtpolitischen Funktion im hellenistischen Raum. Prolegomena zur Typologie der hellenistischen Herrschaftssprache“, G. Brands, - W. Hoepfner (Hg): Basileia. Die Paläste der hellenistischen Könige, Mainz, 1996, 44-55.

32. W. HELD: „Die Residenzstädte der Seleukiden“, JDAI, 117, 2002, pp. 217-249.

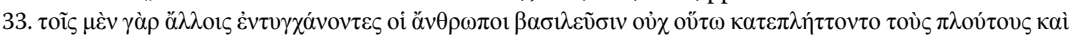

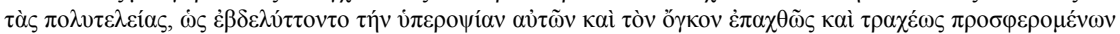

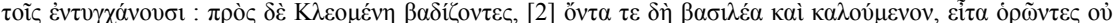

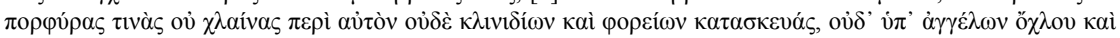

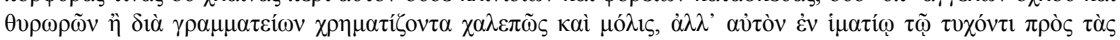

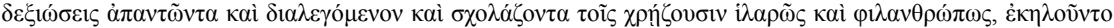

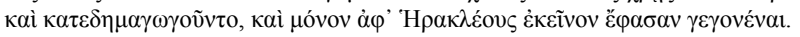


durch Gestik und Mimik. Des Königs Zeit ist begrenzt und er wird permanent abgeschirmt. Das leistet nicht nur subalternes Personal, sondern vor allem der Kreis der Philoi, die als Berater, Vermittler, Gesandte wirken sowie wichtige militärische wie administrative Funktionen übernahmen. Wer im Reich das Ohr des Königs wollte, musste sich um diese Männer bemühen, mußte sie als Makler der Macht anrufen. Der athenische Hymnos auf Demetrios ${ }^{34}$ beschreibt den König von seinen Freunden umgeben und nennt die Philoi die Sterne, den König die Sonne. Die Trabanten besitzen zugegebenermaßen ihren eigenen Glanz, können aber umso heller leuchten, wenn das Hauptgestirn nicht strahlt, an dessen Strahlkraft man leicht zu verbrennen droht. ${ }^{35}$ Gerade Demetrios Poliorketes reglementierte den Zutritt zu seiner Person und versuchte in theatralischer Weise Lebensformen Alexanders zu imitieren. ${ }^{36}$ Von ihm wurde jedoch als König auch erwartet, dass er sich nach makedonischer Tradition persönlich für die Angelegenheiten des Reiches und der Menschen zuständig fühlte. ${ }^{37}$ Die äußerst tendentiöse Darstellung des Demetrios in den Quellen legt nahe, dass er sich von Zeit zu Zeit in der Öffentlichkeit zeigte, um diesen Anforderungen zu genügen, etwa Bittschriften entgegennahm. ${ }^{38}$ Ausssprüche und Geschichten von anderen Königen belegen ebenfalls eine solche Zuständigkeit, ${ }^{39}$ die nicht zuletzt in der gängigen Bezeichnung der Reiche als pragmata tou basiléos fassbar wird.

Eine immer stärkere Ausdifferenzierung der hellenistischen Höfe durch entsprechende Hofränge macht deutlich, dass es nicht makedonische Traditionen waren, die den Ton angaben. Die Könige delegierten vielmehr einen Großteil ihrer Aufgaben an die Philoi, die neben Dynastie und Armee, wie bei den Seleukiden, zur sichtbaren und anerkannten Machtbasis der Monarchie wurden..$^{40}$ Durch das Band der Freundschaft und Verwandtschaft wurde der hohe Einfluss dieser Männer wiederum legitimiert und nicht etwa durch Kompetenz oder Leistungsfähigkeit. In ihrem äußeren Aufwand waren sie gezwungen, sich dem König anzupassen. ${ }^{41}$ Gleichzeitig geschah das Regierungsgeschäft weitestgehend hinter verschlossenen Türen, wenn auch in engster Beratung mit den Freunden. Aber auch ein Philos hatte keinen unbeschränkten Zugang zum König, konnte diesen aber verliehen bekommen. ${ }^{42}$

\footnotetext{
34. DuRIS FGrHist 76 F $13=$ Athen. 6,253b-f.

35. Das Gefolge wird zum unverzichtbaren Bestandteil königlicher Repräsentation. Vgl. die Kritik am Verhalten Antiochos IV. Роцув. 26,11; Diod. 39,27-32

36. Plut. Demet. 43,1.

37. Zur makedon. Tradition vgl. Plut. mor. 179C.

38. Plut. Demet. 42.

39. Zu denken ist an die Beschwerden des ersten Seleukidenherrschers über die umfangreiche königliche Korrespondenz vgl. PLUT. mor. 790A.

40. OGIS 219, Z. 21. Zuletzt R. Strootman: Courts and Elites in the Hellenistic Empires. The Near East After the Achaemenids, c. 330 to 30 BCE, Edinburgh, 2014 (Edinburgh Studies in Ancient Persia), pp. 111ff.; für die Ptolemäer: L. Mooren: La hiérachie de cour ptolémaique. Contribution à létude des institutions et des classes dirigeantes à l'époque héllenistique, Louvain, 1977 (Studia Hellenistica 23).

41. Liv. 45,32,5.

42. LUKIAN SYR. Gotth. 25.
} 
Für Menschen außerhalb dieses Kreises war die königliche Herrschaft jedoch ein Arcanum und fand weitestgehend hinter dem Vorhang statt.

Dennoch wurde dem makedonischen-griechischem Gedanken von Herrschaft durch rituelle Auftritte des Königs in der Öffentlichkeit Rechenschaft getragen. Periodisch öffneten die Herrscher ihre Paläste anläßlich von Feierlichkeiten und ließen zumindest die Bevölkerung der Residenzen teilhaben, oder veranstalteten Prozessionen, bei denen sie sich zeigten. Das läßt sich in Alexandria sehr gut anläßlich der Ptolemaia oder der Adoneia zeigen. Dabei wurde nicht nur die Pracht des Palasts ausgestellt und das Volk bewirtet, sondern auch deutlich gemacht, wer zum vom König bevorzugten Kreis gehörte. ${ }^{43}$ Ptolemaios II. ließ für die Ptolemaia ein Prunkzelt errichten, daran war «an drei Seiten ein gallerieartiger Umgang angelegt, der ein gewölbtes Dach trug. Dort hielt sich das Gefolge der zum Essen geladenen Gäste nach Bedarf auf». ${ }^{44}$ Ein weiterer Aspekt dieser königlichen Auftritte ist der königliche Besuch in den griechischen Poleis, der in ritualisierter Form ablief ${ }^{45}$ und die Distanzierung des Königs aufhob und den Poleis auf diese Weise die Illusion von Selbstbestimmung vermittelte. Ein letztes zu erwähnendes Instrument sind die Einblicke, die der König und seine Familie scheinbar in ihr Privatleben gewährten, sozusagen ein Blick hinter die Kulissen. Die meisten dieser Anekdoten sind so hochgradig konstruiert, dass sie nur erfunden sein können.

43. G. Weber: „Interaktion, Repräsentation und Herrschaft. Der Königshof im Hellenismus“, in: A. Winterling, (Hg.): Zwischen »Haus« und »Staat«. Antike Höfe im Vergleich, München 1997 (Historische Zeitschrift. Beihefte NF 23.), 27-71.

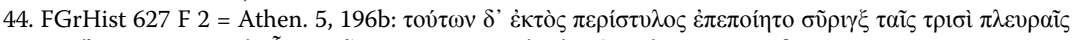

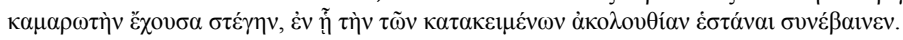

45. R. Strootman: Courts and Elites in the Hellenistic Empires. The Near East After the Achaemenids, c. 330 to 30 BCE, Edinburgh 2014, $47 \mathrm{ff}$. 